\title{
Lightweight Multivariate Sensing in WSNs
}

\author{
João Marco C. Silva ${ }^{1}$, Paulo Carvalho ${ }^{1}$, Kalil Araujo Bispo ${ }^{2}$, and Solange Rito Lima ${ }^{1}$ \\ 1 Centro Algoritmi, Universidade do Minho, 4710-057 Braga, Portugal \\ 2 Dep. de Computação, Universidade Federal de Sergipe, São Cristóvão - SE, 49100-000, Brasil \\ e-mail: \{joaomarco,pmc,solange\}@di.uminho.pt, kalil@dcomp.ufs.br
}

\begin{abstract}
This paper proposes a self-adaptive sampling scheme for WSNs, which aims at capturing accurately the behavior of the physical parameters of interest in each specific WSN context yet reducing the overhead in terms of sensing events. The sampling scheme relies on a set of low-complexity rules capable of auto-regulate the sensing frequency in accordance with each parameter behavior. As proof-of-concept, based on real environmental datasets, we provide statistical indicators illustrating the added value of the proposed sampling scheme in reducing sensing events without compromising the estimation accuracy of physical phenomena.
\end{abstract}

Key words: Sampling techniques; Data sensing; Network measurements; WSNs

\section{Introduction}

In Wireless Sensor Networks (WSNs), the energy consumed by the communication module is a key aspect to be addressed in the network and services design. As discussed in [1], the transmission of a bit throughout the network can consume more energy than processing thousands of instructions, as the communication subsystem has a much higher energy consumption than the computational subsystem. For this reason, communication can be improved through preprocessing the data (e.g., using aggregation schemes) before its dissemination. Other important WSNs characteristic is that the sensing subsystem can lead to a significant source of power consumption and, for this reason, sensing data should be acquired only when necessary, avoiding also unnecessary processing.

In this context, this paper proposes a new adaptive sampling scheme oriented to WSNs aiming at improving the trade off between capturing data accurately and saving energy to enhance operational sensors lifetime. The inherent self-adaptiveness of the sampling scheme provides the required flexibility to be adjusted to distinct sensing scenarios and multiple measuring parameters without human intervention. Through self-regulation of data readings and consequent transmissions, the present work demonstrates that adaptive sampling can be a solid approach to reduce significantly the number of sensing events, while maintaining an accurate view of WSN activity and behavior.

This paper is organized as follows: related work is discussed in Section 2; the sampling scheme rational is described in Section 3; the proof-of-concept and the corresponding evaluation results are discussed in Section 4; and the conclusions are summarized in Section 5. 


\section{Related work}

Although adaptive sampling has been successfully used in conventional computer networks [2,3], the available solutions can hardly be applied in resource constrained networks such as WSNs, due their computational requirements. In [4], the authors propose an optimal sensing scheduling policy for a power capture system equipped with a finite battery. The goal is to select sensing periods strategically so that the average sensing performance is optimized. As performance indicator, the analytical study only considers the battery level of sensors disregarding the variability of the observed parameter. In [5], the authors developed a recovering framework for big data sets through a small number of sensing readings based on space and time correlation characteristics from previous samples. The process lowers the number of transmissions and, consequently, the power consumption in sensor nodes. However, this study is mainly focused on reducing the rate of transmissions and not on the accuracy of the sensing process.

The complex relation between analysis and data acquiring in adaptive sensing paradigms can be extremely powerful, as many times it allows a reliable estimation and signal detection in situations where non-adaptive detection fails [6]. For this reason, the authors investigate a general signal estimation over the adaptive detection paradigm problem, however, they also recognize that the prior knowledge of some required parameters might not be available in a real-life setting. In [7], the authors establish a framework for collecting data from a WSN based on adaptive compressive sensing (as in [5]), taking into account the power consumption and the amount of information in sensing data. Thus, the paper proposes an algorithm to obtain a more precise approximation of the measurements by wasting as less energy as possible. This proposal is also limited to adapt the rate of the transmissions.

From the related literature, most of the studies on adaptive sensing assume either a theoretical approach limiting their applicability to real WSN scenarios or cover a partial set of variables to optimize, such as the rate of transmissions. This evinces the usefulness of studying and improving the trade-off between sensing events and measurements accuracy for distinct WSN contexts. This work is a contribution in this respect.

\section{A sampling scheme for environmental sensing}

This section describes the proposed sampling scheme taking into consideration the heterogeneity of WSN application scenarios and the self-adaptiveness as a mandatory property to assure. In this context, the adaptive sampling scheme uses the temporal variation in the observed scalar physical quantities (e.g., temperature, humidity, etc) in order to self-adjust the interval between two consecutive sensing events. Assuming the likely constraints on sensor devices where the proposed scheme is expected to be deployed, estimating the variation of sampled values resorts to efficient and simple operations, for instance, the lightweight method used in TCP control mechanisms.

Basically, when the sampled values of the observed parameter do not vary significantly, the interval between two sensing events is increased, reducing its frequency, which leads to less computational demand and consequent less energy consumption [1]. Conversely, if a significant variation in the sampled parameter is observed, the time 
scheduling for the next sensing event is decreased in order to improve the accuracy in identifying its temporal fluctuation.

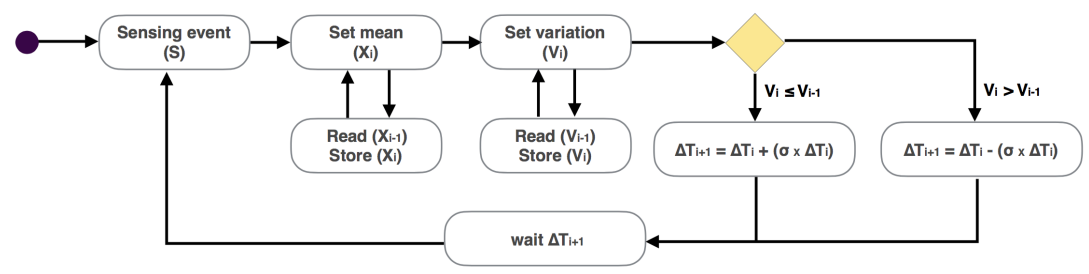

Fig. 1. Adaptive sampling - main steps

In this way, when a sensing event is performed, the mean of the observed variable is calculated using the moving average $\bar{X}_{i}=(1-\eta) \bar{X}_{i-1}+\eta S$, where $\bar{X}_{i-1}$ is the mean calculated in the previous event and $\eta$ is the weight of new observed value $S$. These actions correspond to the initial steps in the flowchart included as Figure 1.

Although the standard deviation is the conventional choice for estimating the variance, it may involve expensive operations to constrained devices related to squaring $\left(\bar{X}_{i}-S\right)$. Alternatively, as discussed in [8], the mean deviation is a good approximation to the standard deviation, being easier to compute as it only resorts to integer arithmetics, even in expressions containing fractions. Therefore, using the current mean value $\bar{X}_{i}$, the variation in the observed values is calculated resorting to the mean deviation $V_{i}=(1-\eta) V_{i-1}+\eta\left|\bar{X}_{i}-S\right|$, where, $V_{i-1}$ is the mean deviation identified in the last sensing event and $\eta$ determines the weight of the current deviation $\left|\bar{X}_{i}-S\right|$.

As illustrated in Figure 1, the adaptive mechanism compares the current estimated variation $\left(V_{i}\right)$ with the variation calculated in the previous sensing event $\left(V_{i-1}\right)$ in order to identify if the observed parameter has changed significantly, and then, the time interval used to schedule the next sensing event is adjusted accordingly. If $V_{i}$ is lower or equal to $V_{i-1}$, the observed parameter did not change significantly from the last sensing events, which allows to reduce the sensing frequency by increasing $\Delta T$. Otherwise, the observed parameter changed significantly since the last observation, which requires more frequent sensing events, obtained by reducing $\Delta T$. An additional constraint is used to prevent $\Delta T$ from growing indefinitely $\left(\Delta T_{\max }\right)$, thus guaranteeing a minimum number of samples per time unit. Conversely, the maximum frequency of sampling $\left(\Delta T_{\min }\right)$ is also limited so that the interval between sensing events does not tend to zero, which would result in an overwhelming resource consumption.

\section{Evaluation results}

The proof-of-concept evaluates the ability of the sampling scheme in: (i) reducing the number of sensing events; (ii) identifying the temporal variation of the observed parameters accurately; and (iii) self-adjusting the sensing frequency in accordance with the variability of environmental parameters. 
The dataset used in the tests was previously collected during approximately six hours, at intervals of five seconds, in a WSN using TelosB motes and publicly available [9]. The WSN consists of eight sensors deployed in indoor and outdoor environments, sensing temperature (indoor - ST1, ST2; outdoor - ST3, ST4) and humidity (indoor $\mathrm{SH} 1, \mathrm{SH} 2$; outdoor - $\mathrm{SH} 3, \mathrm{SH} 4)$. Aiming at evaluating the accuracy in identifying sudden changes in the observed parameters, sensors (ST1, ST4; SH1, SH4) were exposed to a steam of hot water to spark humidity and temperature levels.

The adaptive scheme was set with $\eta=0.7$ in order to stress the weight of the latest sensed value and corresponding variation. The rules mentioned above were set with $\sigma=0.15$, meaning that the interval between samples $(\Delta T)$ is increased by a factor of $15 \%$ when the observed temperature or humidity remain stable, and reduced by a factor of $15 \%$ when the parameter changes significantly. This adjustment may also be indexed to the observed variation or to the current battery level. In this proof-of-concept, $\Delta T_{\max }$ and $\Delta T_{\min }$ were set to 30 and $5 \mathrm{sec}$, respectively.

The performance of the sampling scheme is evaluated relating the number of sensing events along the monitoring period with the accuracy in identifying the temporal variation of the observed parameters (temperature and humidity). A reduction in the number of sensing events leads to less computational operations and, consequently, less energy consumption, however, this reduction should not compromise the accurate observation of physical parameters under monitoring.

As deterministic and adaptive sampling mechanisms yield distinct number of samples, the statistical analysis considers the mean estimated value per second from the resultant time series. The accuracy is then estimated resorting to the Mean Squared Error (MSE), which is commonly used to evaluate the accuracy of estimators, and to the correlation between the time series.

Regarding the ability to reduce the number of sensing events, Figure 2 shows that, for all nodes and parameters, the adaptive scheme only performs around $20 \%$ of the events observed through the deterministic scheme. Attending to the lightweight algorithm ruling the proposed scheme, this significant reduction will also contribute to reduce energy consumption in the sensors.
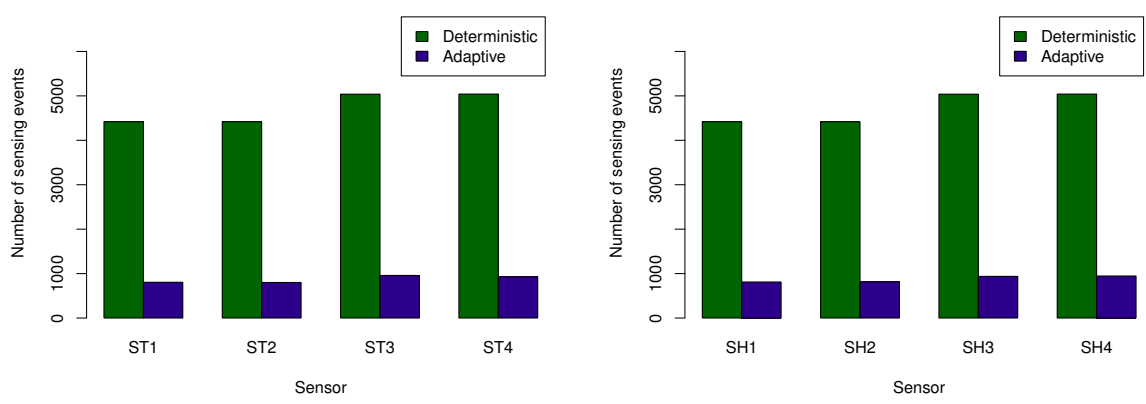

Fig. 2. Number of sensing events: Temperature (left); Humidity (right) 
However, despite of reducing the number of sensing events significantly, proving the efficiency of the adaptive scheme requires verifying its ability in capturing the real distribution of the observed parameters. This is accomplished by estimating the statistical representativeness of adaptive time series against the deterministic behavior. In this way, Figures 3 and 4 show the distribution of sampled values along the test period, for indoor and outdoor sensors. The almost complete overlap of adaptive and deterministic resultant series, highlighted during both smooth and unstable periods, demonstrates that, even reducing the sensing events in around $80 \%$, the adaptive scheme has the capability to catch up the real parameter pattern. Note that, even in presence of sudden environmental changes affecting sensors ST1, ST4, SH1 and SH4 (detailed above), the adaptive scheme adjusts the sensing schedule correctly, confirming the versatility of the self-adaptive scheme.

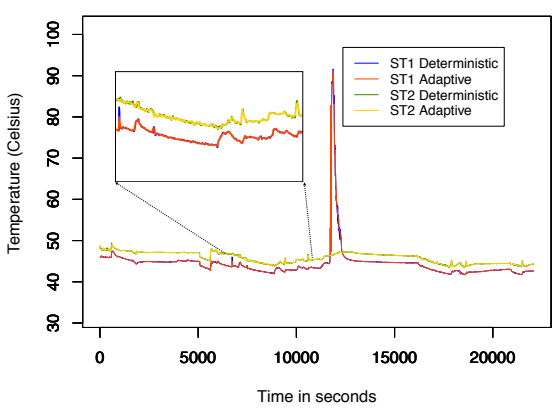

(a) Indoor sensors.

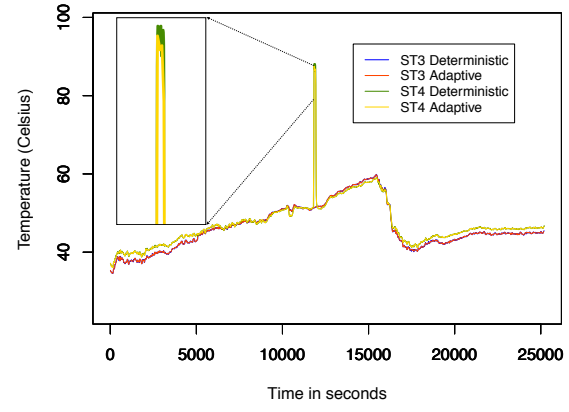

(b) Outdoor sensors.

Fig. 3. Temperature observations.

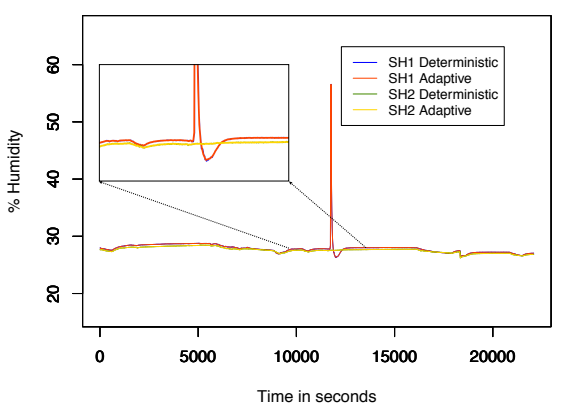

(a) Indoor sensors.

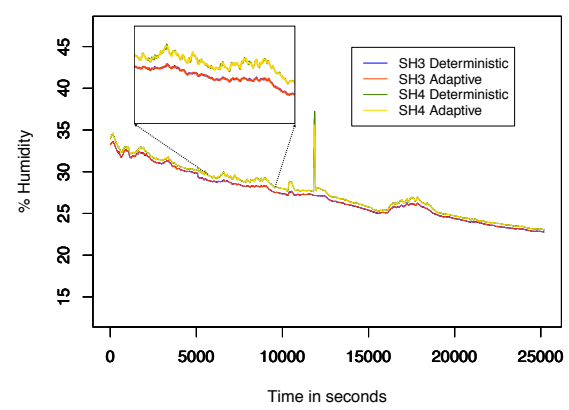

(b) Outdoor sensors.

Fig. 4. Humidity observations. 
These observations are corroborated through the high correlation ( 0.98 for all series) between the distributions of estimated parameters, and the low MSE (approximately 0.5 for temperature and 0.03 for humidity). This means that, for the considered scenarios, the estimation of humidity levels was more accurate, however, as depicted in Figure 3, the temperature was also accurately estimated. The differences in accuracy can however be explained with the use of a constant adjustment factor $\sigma$. In this regard, future work will focus on evolving the mechanism by indexing this factor to the estimated variation in observed parameter.

\section{Conclusions}

Attending to the inherent resource constraints of WSNs, this paper has proposed a selfadaptive sampling scheme able to capture the behavior of multiple physical parameters accurately while reducing the overhead of sensing events and, consequently, the levels of energy consumption. The ability to adapt the sampling frequency through low-cost algorithms was also defined as a design goal, due to the well known low-processing capabilities of sensor nodes. The proof-of-concept has provided initial results attesting the proposal ability to measure environmental parameters accurately, while reducing in $80 \%$ the number of sensing events comparing to deterministic sampling.

Acknowledgements This work has been supported by COMPETE: POCI-01-0145FEDER-007043 and FCT - Fundação para a Ciência e Tecnologia within the Project Scope: UID/CEC/00319/2013.

\section{References}

1. Anastasi, G., Conti, M., Di Francesco, M., Passarella, A.: Energy conservation in wireless sensor networks: A survey. Ad Hoc Networks 7(3) (2009) 537-568

2. Hernandez, E.A., Chidester, M.C., George, A.D.: Adaptive Sampling for Network Management. Journal of Network and Systems Management 9(4) (2001) 409-434

3. Silva, J.M.C., Carvalho, P., Rito Lima, S.: A multiadaptive sampling technique for costeffective network measurements. Computer Networks 57(17) (December 2013) 3357-3369

4. Yang, J., Wu, X., Wu, J.: Adaptive sensing scheduling for energy harvesting sensors with finite battery. In: IEEE International Conference on Communications (ICC). (June 2015) 98-103

5. Quer, G., Masiero, R., Pillonetto, G., Rossi, M., Zorzi, M.: Sensing, compression, and recovery for wsns: Sparse signal modeling and monitoring framework. IEEE Transactions on Wireless Communications 11(10) (October 2012) 3447-3461

6. Castro, R.M., Tanczos, E.: Adaptive sensing for estimation of structured sparse signals. IEEE Transactions on Information Theory 61(4) (April 2015) 2060-2080

7. Chou, C.T., Rana, R., Hu, W.: Energy efficient information collection in wireless sensor networks using adaptive compressive sensing. In: 2009 IEEE 34th Conference on Local Computer Networks. (Oct 2009) 443-450

8. Jacobson, V.: Congestion avoidance and control. In: Symposium Proc. on Communications Architectures and Protocols. SIGCOMM '88, New York, NY, USA, ACM (1988) 314-329

9. Suthaharan, S., Alzahrani, M., Rajasegarar, S., Leckie, C., Palaniswami, M.: Labelled data collection for anomaly detection in wireless sensor networks. In: Intelligent sensors, sensor networks and information processing (ISSNIP), 6th Int. Conference on, IEEE (2010) 269-274 